\title{
FACTORIZATION THEOREMS FOR GENERALIZED LAMBERT SERIES AND APPLICATIONS
}

\author{
MIRCEA MERCA \\ ACADEMY OF ROMANIAN SCIENTISTS \\ SPLAIUL INDEPENDENTEI 54, BUCHAREST, 050094 ROMANIA \\ MIRCEA.MERCA@PROFINFO.EDU.RO
}

\author{
MAXIE D. SCHMIDT \\ SCHOOL OF MATHEMATICS \\ GEORGIA INSTITUTE OF TECHNOLOGY \\ ATLANTA, GA 30332 USA \\ MAXIEDS@GMAIL.COM \\ MSCHMIDT34@GATECH.EDU
}

\begin{abstract}
We prove new variants of the Lambert series factorization theorems studied by Merca and Schmidt (2017) which correspond to a more general class of Lambert series expansions of the form $L_{a}(\alpha, \beta ; q):=\sum_{n \geq 1} a_{n} q^{\alpha n-\beta} /(1-$ $q^{\alpha n-\beta}$ ) for integers $\alpha, \beta$ defined such that $\alpha \geq 1$ and $0 \leq \beta<\alpha$. Applications of the new results in the article are given to restricted divisor sums over several classical special arithmetic functions which define the cases of well-known, so-termed "ordinary" Lambert series expansions cited in the introduction. We prove several new forms of factorization theorems for Lambert series over a convolution of two arithmetic functions which similarly lead to new applications relating convolutions of special multiplicative functions to partition functions and $n$-fold convolutions of one of the special functions.
\end{abstract}

\section{INTRODUCTION}

1.1. Factorizations of generalized Lambert series. For fixed $\alpha, \beta \in \mathbb{Z}$ such that $\alpha \geq 1$ and $0 \leq \beta<\alpha$, and an arbitrary sequence $\left\{a_{n}\right\}_{n \geq 1}$, we consider generalized Lambert series expansions of the form

$$
L_{a}(\alpha, \beta ; q):=\sum_{n \geq 1} \frac{a_{n} q^{\alpha n-\beta}}{1-q^{\alpha n-\beta}}=\sum_{m \geq 1} b_{m} \cdot q^{m},\left|q^{\alpha}\right|<1 .
$$

The coefficients of the generalized Lambert series expansion on the left-hand-side of the previous equation are given by

$$
b_{m}=\sum_{\alpha d-\beta \mid m} a_{d}
$$

Several well known variants of the ordinary Lambert series expansions studied in $[4,5,6,8]$ which generate special arithmetic functions are transformed into a series of the form in (1) as follows where $\mu(n)$ denotes the Möbius function, $\phi(n)$ denotes

Date: Wednesday $12^{\text {th }}$ September, 2018.

2010 Mathematics Subject Classification. 11A25; 11P81; 05A17; 05A19.

Key words and phrases. Lambert series; factorization theorem; matrix factorization; partition function; multiplicative function. 
Euler's totient function, $\sigma_{\alpha}(n)$ denotes the generalized sum of divisors function for a fixed $\alpha \in \mathbb{C}, \lambda(n)$ denotes Liouville's function, $\Lambda(n)$ denotes von Mangoldt's function, $\omega(n)$ defines the number of distinct primes dividing $n$, and $J_{t}(n)$ is Jordan's totient function for some fixed $t \in \mathbb{C}[7, \S 27][4, c f . \S 1, \S 3]$ :

$$
\begin{aligned}
& \sum_{n \geq 1} \frac{\mu(n) q^{\alpha n-\beta}}{1-q^{\alpha n-\beta}}=\sum_{m \geq 1} \sum_{\alpha d-\beta \mid m} \mu(d) \cdot q^{m} \\
& \sum_{n \geq 1} \frac{\phi(n) q^{\alpha n-\beta}}{1-q^{\alpha n-\beta}}=\sum_{m \geq 1} \sum_{\alpha d-\beta \mid m} \phi(d) \cdot q^{m} \\
& \sum_{n \geq 1} \frac{n^{x} q^{\alpha n-\beta}}{1-q^{\alpha n-\beta}}=\sum_{m \geq 1} \sum_{\alpha d-\beta \mid m} d^{x} \cdot q^{m} \\
& \sum_{n \geq 1} \frac{\lambda(n) q^{\alpha n-\beta}}{1-q^{\alpha n-\beta}}=\sum_{m \geq 1} \sum_{\alpha d-\beta \mid m} \lambda(d) \cdot q^{m} \\
& \sum_{n \geq 1} \frac{\Lambda(n) q^{\alpha n-\beta}}{1-q^{\alpha n-\beta}}=\sum_{m \geq 1} \sum_{\alpha d-\beta \mid m} \Lambda(d) \cdot q^{m} \\
& \sum_{n \geq 1} \frac{|\mu(n)| q^{\alpha n-\beta}}{1-q^{\alpha n-\beta}}=\sum_{m \geq 1} \sum_{\alpha d-\beta \mid m}|\mu(d)| \cdot q^{m} \\
& \sum_{n \geq 1} \frac{J_{t}(n) q^{\alpha n-\beta}}{1-q^{\alpha n-\beta}}=\sum_{m \geq 1} \sum_{\alpha d-\beta \mid m} J_{t}(d) \cdot q^{m} .
\end{aligned}
$$

Moreover, in the special case where $(\alpha, \beta):=(2,1)$, we have another Lambert series expansion generating the sum of squares function, $r_{2}(n)$, of the form $[2, \S 17.10]$

$$
\sum_{m \geq 1} r_{2}(m) q^{m}=\sum_{n \geq 1} \frac{4 \cdot(-1)^{n+1} q^{2 n-1}}{1-q^{2 n-1}} .
$$

For the remainder of the article we treat the generalized series parameters $\alpha, \beta$ to be defined by the constraints above and the sequence $\left\{a_{n}\right\}_{n \geq 1}$ to be arbitrary unless otherwise specified.

Within this article, we extend the so-termed "factorization theorems" proved in $[4,5,6,8]$ to the generalized Lambert series cases defined in (1). In particular, we consider factorizations of the form

$$
L_{a}(\alpha, \beta ; c q)=\frac{1}{C(q)} \sum_{n \geq 1} \sum_{k=1}^{n} s_{n, k} \bar{a}_{k}(c q)^{n},
$$

where $\bar{a}_{n}$ depends only on the $s_{n, k}$ and on the sequence of $a_{n}$. In general, when $\alpha>1$ and $\bar{a}_{n} \equiv a_{n}$ for all $n \geq 1$, we typically see that the square matrices, $A_{n}:=\left(s_{i, j}\right)_{1 \leq i, j \leq n}$, are not invertible as in the cases of the first factorization theorems proved in the references $[4,5,8]$. However, we may still proceed to define an inverse sequence, $s_{n, k}^{(-1)}$, as in the references with a corresponding non-singular matrix representation which implicitly defines the sequence of $s_{n, k}$ as in [5] for $\bar{a}_{n} / \equiv a_{n}$. In these cases, we have a matrix representation of the factorization 
theorem in (3) of the form

$$
\left[\begin{array}{c}
\bar{a}_{1} \\
\bar{a}_{2} \\
\vdots \\
\bar{a}_{n}
\end{array}\right]=A_{n}^{-1}\left[\begin{array}{c}
B_{0} \\
B_{1} \\
\vdots \\
B_{n-1}
\end{array}\right],
$$

where the sequence of $\left\{B_{m}\right\}_{m \geq 0}$ depends on the arithmetic function, $b_{m}$, implicit to the expansion of (1) and the factorization pair, $\left(C(q), s_{n, k}\right)$. When the matrix $A_{n}$ is non-singular, we have the next determinant-based recurrence relations proved as in $[4, \S 2]$ relating the two sequences, $s_{n, k}$ and $s_{n, k}^{(-1)}$.

$$
\begin{aligned}
s_{n, j}^{(-1)} & =-\sum_{k=1}^{n-j} s_{n, n+1-k}^{(-1)} \cdot s_{n+1-k, j}+\delta_{n, j} \\
& =-\sum_{k=1}^{n-j} s_{n, n-k} \cdot s_{n-k, j}^{(-1)}+\delta_{n, j}
\end{aligned}
$$

These identities are symmetric in that these identities still hold if one sequence is interchanged with the other.

1.2. Significance of our new results. In this article, we prove a few variants corresponding to the expansions of (1) which effectively generalize the Lambert series factorization theorems found in the first references [4,5]. Namely, we prove the key results in Theorem 2.5 and Theorem 3.1 and their corollaries which provide analogous factorization theorems for the generalized cases of the Lambert series defined in the first section of the introduction above. As in the similar and closely-related factorization results found in the references, each of these factorization theorems provide new relations between sums over an arbitrary function, $a_{n}$, involving the divisors of $n$ and more additive identities involving partition functions and the same function $a_{n}$. Thus the results proved in this article continue the spirit of $[4,5,6,8]$ by connecting the at times seemingly disparate branches of additive and multiplicative number theory in new and interesting ways.

Central to the importance of our new results are the applications to modified divisors sums for many classical special arithmetic functions often studied in additive and multiplicative number theory. These special functions include the Möbius function $\mu(n)$, Euler's totient function $\phi(n)$, the sum-of-divisors functions $\sigma_{\alpha}(n)$, Liouville's function $\lambda(n)$, von Mangoldt's function $\Lambda(n),|\mu(n)|$, the number of distinct primes dividing $n, \omega(n)$, Jordan's totient function $J_{t}(n)$, and the sum of squares function $r_{2}(n)$. In particular, we provide a number of examples of our new results pertaining to these classical special functions in Section 2 and Section 3. Also discussed in this article are conjectures on the expansions of degenerate cases of Theorem 2.5 given in terms of nested formulas involving Euler's partition function $p(n)$ (see Remark 2.7). These conjectured identities are interesting in form for their own sake, and fit in with the properties we establish for the generalized Lambert series cases in (1).

Finally, we conclude the article by stating and proving another somewhat more general class of factorization theorems for Lambert series over a convolution of arithmetic functions, $f * g$. These uniquely new results lead to still more applications connecting partition functions and special functions from multiplicative 
number theory. In particular, in Section 4 of the article we prove Proposition 4.1 and Theorem 4.2 providing the more difficult to obtain corresponding forms of the inverse sequences specifying these factorizations. Furthermore, the remarks given in Section 5.3 offer several concluding suggestions on enumerating new, and more general factorization theorems for the expansions of Lambert series generating functions which we have so far not considered in this article or in the references. Our continued aim in exploring these new variations of the Lambert series factorization theorems is to branch out and provide further connections between the inherently multiplicative structure of the Lambert series generating functions and the additive theory of partitions and special partition functions.

\section{Generalized Factorization Theorems}

2.1. Special Cases. Before we state and prove the generalized factorization theorem results in the following subsections, we first consider the special case series expansions identified below. These next results given in this subsection identify several new interpretations of the factor pair sequence $s_{n, k}$ as well as provide generalized analogs to the expansions of several identities cited in [5].

Proposition 2.1. For $|q|<1$, we have that

$$
\sum_{n=1}^{\infty} a_{n} \frac{q^{2 n-1}}{1-q^{2 n-1}}=\frac{1}{\left(q ; q^{2}\right)_{\infty}} \sum_{n=1}^{\infty}\left(\sum_{k=1}^{n} s_{n, k} a_{k}\right)(-1)^{n-1} q^{n},
$$

where $s_{n, k}$ denotes the number of $(2 k-1)$ 's in all partitions of $n$ into distinct odd parts.

Proof. We consider the identity [6, eq. 2.1], namely

$\sum_{k=1}^{n} \frac{a_{k} x_{k}}{1-x_{k}}=\left(\prod_{k=1}^{n} \frac{1}{1-x_{k}}\right)\left(\sum_{k=1}^{n} \sum_{1 \leq i_{1}<\ldots<i_{k} \leq n}(-1)^{k-1}\left(a_{i_{1}}+\cdots+a_{i_{k}}\right) x_{i_{1}} \cdots x_{i_{k}}\right)$.

By this relation with $x_{k}$ replaced by $q^{2 k-1}$, we get

$$
\begin{aligned}
& \sum_{k=1}^{n} \frac{a_{k} q^{2 k-1}}{1-q^{2 k-1}} \\
& \quad=\frac{1}{\left(q ; q^{2}\right)_{n}}\left(\sum_{k=1}^{n} \sum_{1 \leq i_{1}<\ldots<i_{k} \leq n}(-1)^{k-1}\left(a_{i_{1}}+\cdots+a_{i_{k}}\right) q^{\left(2 i_{1}-1\right)+\cdots+\left(2 i_{k}-1\right)}\right) .
\end{aligned}
$$

The result follows directly from this identity considering the limiting case as $n \rightarrow$ $\infty$.

Example 2.2 (Consequences of the Proposition). The result in Proposition 2.1 allows us to derive many special case identities involving Euler's partition function and various arithmetic functions. More precisely, by the well-known famous special cases Lambert series identities expanded in the introduction to [4], for $n \geq 1$ we have that

$$
\sum_{k=1}^{n} \sum_{2 d-1 \mid k} d^{x} \cdot \widetilde{q}(n-k)=\sum_{k=1}^{n}(-1)^{n-1} k^{x} s_{n, k}
$$




$$
\begin{aligned}
& \sum_{k=1}^{n} \sum_{2 d-1 \mid k} \mu(d) \cdot \widetilde{q}(n-k)=\sum_{k=1}^{n}(-1)^{n-1} \mu(k) s_{n, k}, \\
& \sum_{k=1}^{n} \sum_{2 d-1 \mid k} \phi(d) \cdot \widetilde{q}(n-k)=\sum_{k=1}^{n}(-1)^{n-1} \phi(k) s_{n, k}, \\
& \sum_{k=1}^{n} \sum_{2 d-1 \mid k} \lambda(d) \cdot \widetilde{q}(n-k)=\sum_{k=1}^{n}(-1)^{n-1} \lambda(k) s_{n, k}, \\
& \sum_{k=1}^{n} \sum_{2 d-1 \mid k} \log (d) \cdot \widetilde{q}(n-k)=\sum_{k=1}^{n}(-1)^{n-1} \log (k) s_{n, k}, \\
& \sum_{k=1}^{n} \sum_{2 d-1 \mid k}|\mu(d)| \cdot \widetilde{q}(n-k)=\sum_{k=1}^{n}(-1)^{n-1}|\mu(k)| s_{n, k}, \\
& \sum_{k=1}^{n} \sum_{2 d-1 \mid k} J_{t}(d) \cdot \widetilde{q}(n-k)=\sum_{k=1}^{n}(-1)^{n-1} J_{t}(k) s_{n, k},
\end{aligned}
$$

where $s_{n, k}$ is the number of $(2 k-1)$ 's in all partitions of $n$ into distinct odd parts and $\widetilde{q}(n):=s_{e}(n)-s_{o}(n)$ where $s_{e}(n)$ and $s_{o}(n)$ respectively denote the numbers of partitions of $n$ into even (odd) parts. We can also similarly express the relations in the previous equations for any special arithmetic function $a_{n}$ in the form of

$$
\sum_{2 d-1 \mid n} a_{d}=\sum_{k=0}^{n} \sum_{j=1}^{k}(-1)^{k-1} q(n-k) s_{k, j} a_{j}
$$

where the partition function $q(n)$ denotes the number partitions of $n$ into (distinct) odd parts. Moreover, since we have a direct factorization of the Lambert series generating function for the sum of squares function in the form of the proposition, we may write

$$
\sum_{k=1}^{n} r_{2}(k) \widetilde{q}(n-k)=\sum_{k=1}^{n} 4 \cdot(-1)^{k+1} s_{n, k}
$$

using the same notation as above. Similarly, we expand $r_{2}(n)$ as the multiple sum

$$
r_{2}(n)=\sum_{k=0}^{n} \sum_{j=1}^{k} 4 \cdot q(n-k)(-1)^{j+1} s_{k, j}
$$

for all $n \geq 1$.

Proposition 2.3. For $|q|<1,0 \leq \beta<\alpha$,

$$
\sum_{n=1}^{\infty} a_{n} \frac{q^{\alpha n-\beta}}{1-q^{\alpha n-\beta}}=\frac{1}{\left(q^{\alpha-\beta} ; q^{\alpha}\right)_{\infty}} \sum_{n=1}^{\infty}\left(\sum_{k=1}^{n}\left(s_{o}(n, k)-s_{e}(n, k)\right) a_{k}\right) q^{n},
$$

where $s_{o}(n, k)$ and $s_{e}(n, k)$ denotes the number of $(\alpha k-\beta)$ 's in all partition of $n$ into odd (even) number of distinct parts of the form $\alpha k-\beta$.

Proof. The proof follows from [6, eq. 2.1], replacing $x_{k}$ by $q^{\alpha k-\beta}$. 
Proposition 2.4. For $|q|<1,0 \leq \beta<\alpha$,

$$
\sum_{n=1}^{\infty} a_{n} \frac{q^{\alpha n-\beta}}{1-q^{\alpha n-\beta}}=\left(q^{\alpha-\beta} ; q^{\alpha}\right)_{\infty} \sum_{n=1}^{\infty}\left(\sum_{k=1}^{n} s(n, k) a_{k}\right) q^{n},
$$

where $s(n, k)$ denotes the number of $(\alpha k-\beta)$ 's in all partition of $n$ into parts of the form $\alpha k-\beta$.

Proof. We take into account the fact that

$$
\frac{q^{\alpha n-\beta}}{1-q^{\alpha n-\beta}} \cdot \frac{1}{\left(q^{\alpha-\beta} ; q^{\alpha}\right)_{\infty}}
$$

is the generating function for the number of $(\alpha k-\beta)$ 's in all partitions of $n$ into parts of the form $\alpha k-\beta$. This generating function implies our result.

\subsection{The first generalized factorization theorem.}

Theorem 2.5 (A General Formula for $s_{n, k}$ ). For fixed $\alpha, \beta, \gamma, \delta \in \mathbb{Z}$ such that $\alpha, \gamma \geq 1,1 \leq \beta<\alpha$, and $1 \leq \delta<\gamma$, the factorization pair $\left(C(q), s_{n, k}\right)$ in the generalized Lambert series factorization expanded by

$$
L_{a}(\alpha, \beta, \gamma, \delta ; q):=\sum_{n \geq 1} \frac{a_{n} q^{\alpha n+\beta}}{1-q^{\gamma n+\delta}}=\frac{1}{C(q)} \sum_{n \geq 1} \sum_{k=1}^{n} s_{n, k} a_{k} \cdot q^{n},
$$

satisfies

$$
s_{n, k}=\left[q^{n}\right] \frac{q^{\alpha n+\beta}}{1-q^{\gamma n+\delta}} C(q) .
$$

Specific interpretations of the sequence $s_{n, k}$ are given as special cases of the previous expansions as in the results proved in the last subsection.

Proof. We begin by rewriting (i) in the form of

$$
C(q) \sum_{k \geq 1} \frac{a_{k} q^{\alpha k+\beta}}{1-q^{\gamma k+\delta}}=\sum_{k \geq 1}\left(\sum_{n \geq 1} s_{n, k} q^{n}\right) a_{k} .
$$

Then if we equate the coefficients of $a_{k}$ in the previous equation, we see that

$$
C(q) \frac{q^{\alpha k+\beta}}{1-q^{\gamma k \delta}}=\sum_{n \geq 1} s_{n, k} q^{n},
$$

which implies our stated result.

Corollary 2.6. Let $\alpha \geq 1$ and $0 \leq \beta<\alpha$ be integers and suppose that $\delta \in \mathbb{Z}$. Suppose that

$$
\sum_{n \geq 1} \frac{a_{n} q^{n}}{1-q^{n}}=\frac{1}{C(q)} \sum_{n \geq 0} \sum_{k=1}^{n} s_{n, k} a_{k} \cdot q^{n}
$$

and that

$$
\sum_{n \geq 1} \frac{a_{n} q^{\alpha n-\beta+\delta}}{1-q^{\alpha n-\beta}}=\frac{1}{C(q)} \sum_{n \geq 0} \sum_{k=1}^{n} s_{n, k}(\alpha, \beta ; \delta) a_{k} \cdot q^{n} .
$$

Then we have that

$$
s_{n, k}(\alpha, \beta ; \delta)=s_{n-\delta, \alpha k-\beta} .
$$




\begin{tabular}{|cccccccccccccccc|}
\hline 1 & 0 & 0 & 0 & 0 & 0 & 0 & 0 & 0 & 0 & 0 & 0 & 0 & 0 & 0 & 0 \\
-1 & 1 & 0 & 0 & 0 & 0 & 0 & 0 & 0 & 0 & 0 & 0 & 0 & 0 & 0 & 0 \\
-1 & -1 & 1 & 0 & 0 & 0 & 0 & 0 & 0 & 0 & 0 & 0 & 0 & 0 & 0 & 0 \\
1 & -1 & -1 & 1 & 0 & 0 & 0 & 0 & 0 & 0 & 0 & 0 & 0 & 0 & 0 & 0 \\
-1 & 0 & -1 & -1 & 1 & 0 & 0 & 0 & 0 & 0 & 0 & 0 & 0 & 0 & 0 & 0 \\
0 & 0 & 0 & -1 & -1 & 1 & 0 & 0 & 0 & 0 & 0 & 0 & 0 & 0 & 0 & 0 \\
1 & 2 & 0 & 0 & -1 & -1 & 1 & 0 & 0 & 0 & 0 & 0 & 0 & 0 & 0 & 0 \\
0 & -1 & 1 & 0 & 0 & -1 & -1 & 1 & 0 & 0 & 0 & 0 & 0 & 0 & 0 & 0 \\
0 & 0 & 0 & 1 & 0 & 0 & -1 & -1 & 1 & 0 & 0 & 0 & 0 & 0 & 0 & 0 \\
1 & 0 & 2 & 0 & 1 & 0 & 0 & -1 & -1 & 1 & 0 & 0 & 0 & 0 & 0 & 0 \\
0 & 0 & -1 & 1 & 0 & 1 & 0 & 0 & -1 & -1 & 1 & 0 & 0 & 0 & 0 & 0 \\
0 & 2 & -1 & 0 & 1 & 0 & 1 & 0 & 0 & -1 & -1 & 1 & 0 & 0 & 0 & 0 \\
0 & -1 & 0 & 1 & 0 & 1 & 0 & 1 & 0 & 0 & -1 & -1 & 1 & 0 & 0 & 0 \\
0 & -1 & 0 & -1 & 0 & 0 & 1 & 0 & 1 & 0 & 0 & -1 & -1 & 1 & 0 & 0 \\
0 & 0 & 0 & -1 & 0 & 0 & 0 & 1 & 0 & 1 & 0 & 0 & -1 & -1 & 1 & 0 \\
-1 & 0 & 0 & -1 & 1 & 0 & 0 & 0 & 1 & 0 & 1 & 0 & 0 & -1 & -1 & 1 \\
\hline
\end{tabular}

(i) $s_{n, k}$

\begin{tabular}{|cccccccccccccccc|}
\hline 1 & 0 & 0 & 0 & 0 & 0 & 0 & 0 & 0 & 0 & 0 & 0 & 0 & 0 & 0 & 0 \\
1 & 1 & 0 & 0 & 0 & 0 & 0 & 0 & 0 & 0 & 0 & 0 & 0 & 0 & 0 & 0 \\
2 & 1 & 1 & 0 & 0 & 0 & 0 & 0 & 0 & 0 & 0 & 0 & 0 & 0 & 0 & 0 \\
2 & 2 & 1 & 1 & 0 & 0 & 0 & 0 & 0 & 0 & 0 & 0 & 0 & 0 & 0 & 0 \\
5 & 3 & 2 & 1 & 1 & 0 & 0 & 0 & 0 & 0 & 0 & 0 & 0 & 0 & 0 & 0 \\
7 & 5 & 3 & 2 & 1 & 1 & 0 & 0 & 0 & 0 & 0 & 0 & 0 & 0 & 0 & 0 \\
9 & 6 & 5 & 3 & 2 & 1 & 1 & 0 & 0 & 0 & 0 & 0 & 0 & 0 & 0 & 0 \\
15 & 11 & 7 & 5 & 3 & 2 & 1 & 1 & 0 & 0 & 0 & 0 & 0 & 0 & 0 & 0 \\
22 & 15 & 11 & 7 & 5 & 3 & 2 & 1 & 1 & 0 & 0 & 0 & 0 & 0 & 0 & 0 \\
27 & 21 & 14 & 11 & 7 & 5 & 3 & 2 & 1 & 1 & 0 & 0 & 0 & 0 & 0 & 0 \\
42 & 30 & 22 & 15 & 11 & 7 & 5 & 3 & 2 & 1 & 1 & 0 & 0 & 0 & 0 & 0 \\
55 & 41 & 30 & 22 & 15 & 11 & 7 & 5 & 3 & 2 & 1 & 1 & 0 & 0 & 0 & 0 \\
74 & 54 & 41 & 29 & 22 & 15 & 11 & 7 & 5 & 3 & 2 & 1 & 1 & 0 & 0 & 0 \\
101 & 77 & 56 & 42 & 30 & 22 & 15 & 11 & 7 & 5 & 3 & 2 & 1 & 1 & 0 & 0 \\
135 & 101 & 77 & 56 & 42 & 30 & 22 & 15 & 11 & 7 & 5 & 3 & 2 & 1 & 1 & 0 \\
170 & 132 & 99 & 76 & 55 & 42 & 30 & 22 & 15 & 11 & 7 & 5 & 3 & 2 & 1 & 1 \\
\hline
\end{tabular}

(ii) $s_{n, k}^{(-1)}$

\begin{tabular}{|cccccccccccccccc|}
\hline 1 & 0 & 0 & 0 & 0 & 0 & 0 & 0 & 0 & 0 & 0 & 0 & 0 & 0 & 0 & 0 \\
0 & 1 & 0 & 0 & 0 & 0 & 0 & 0 & 0 & 0 & 0 & 0 & 0 & 0 & 0 & 0 \\
0 & 1 & 1 & 0 & 0 & 0 & 0 & 0 & 0 & 0 & 0 & 0 & 0 & 0 & 0 & 0 \\
-1 & 1 & 1 & 1 & 0 & 0 & 0 & 0 & 0 & 0 & 0 & 0 & 0 & 0 & 0 & 0 \\
0 & 3 & 2 & 1 & 1 & 0 & 0 & 0 & 0 & 0 & 0 & 0 & 0 & 0 & 0 & 0 \\
0 & 2 & 2 & 2 & 1 & 1 & 0 & 0 & 0 & 0 & 0 & 0 & 0 & 0 & 0 & 0 \\
-2 & 6 & 5 & 3 & 2 & 1 & 1 & 0 & 0 & 0 & 0 & 0 & 0 & 0 & 0 & 0 \\
1 & 7 & 6 & 4 & 3 & 2 & 1 & 1 & 0 & 0 & 0 & 0 & 0 & 0 & 0 & 0 \\
0 & 13 & 9 & 7 & 5 & 3 & 2 & 1 & 1 & 0 & 0 & 0 & 0 & 0 & 0 & 0 \\
-3 & 13 & 12 & 10 & 6 & 5 & 3 & 2 & 1 & 1 & 0 & 0 & 0 & 0 & 0 & 0 \\
0 & 30 & 22 & 15 & 11 & 7 & 5 & 3 & 2 & 1 & 1 & 0 & 0 & 0 & 0 & 0 \\
1 & 27 & 23 & 18 & 14 & 10 & 7 & 5 & 3 & 2 & 1 & 1 & 0 & 0 & 0 & 0 \\
-3 & 54 & 41 & 29 & 22 & 15 & 11 & 7 & 5 & 3 & 2 & 1 & 1 & 0 & 0 & 0 \\
2 & 60 & 51 & 39 & 28 & 21 & 14 & 11 & 7 & 5 & 3 & 2 & 1 & 1 & 0 & 0 \\
0 & 90 & 68 & 54 & 40 & 30 & 22 & 15 & 11 & 7 & 5 & 3 & 2 & 1 & 1 & 0 \\
-4 & 107 & 90 & 69 & 52 & 40 & 29 & 21 & 15 & 11 & 7 & 5 & 3 & 2 & 1 & 1 \\
\hline
\end{tabular}

(iii) $\gamma_{k}(n)$

Figure 2.1. A generalized factorization for $L_{a}(1,0,2,1 ; q)$

In particular, when $C(q) \equiv(q ; q)_{\infty}$ we have that the result in the equation above holds for $s_{n, k}=s_{o}(n, k)-s_{e}(n, k)$ where $s_{o}(n, k)$ and $s_{e}(n, k)$ are respectively the number of $k$ 's in all partitions of $n$ into an odd (even) number of distinct parts.

Proof. The proof follows from the second statement in the theorem which provides a generating function for $s_{n, k}$ for all $n, k \geq 1$.

Remark 2.7 (Conjectures on Degenerate Cases of the Theorem). One distinction to be made between the factorization result in the theorem and the results in $[4,5]$ 


\begin{tabular}{|cccccccccc|}
\hline 1 & 0 & 0 & 0 & 0 & 0 & 0 & 0 & 0 & 0 \\
-1 & 1 & 0 & 0 & 0 & 0 & 0 & 0 & 0 & 0 \\
-1 & -1 & 1 & 0 & 0 & 0 & 0 & 0 & 0 & 0 \\
$d$ & -1 & -1 & 1 & 0 & 0 & 0 & 0 & 0 & 0 \\
$-d$ & 0 & -1 & -1 & 1 & 0 & 0 & 0 & 0 & 0 \\
$1-d$ & 0 & 0 & -1 & -1 & 1 & 0 & 0 & 0 & 0 \\
$d^{2}$ & $d+1$ & 0 & 0 & -1 & -1 & 1 & 0 & 0 & 0 \\
$1-d^{2}$ & $-d$ & 1 & 0 & 0 & -1 & -1 & 1 & 0 & 0 \\
$d-d^{2}$ & $1-d$ & 0 & 1 & 0 & 0 & -1 & -1 & 1 & 0 \\
$d^{3}$ & 0 & $d+1$ & 0 & 1 & 0 & 0 & -1 & -1 & 1 \\
\hline
\end{tabular}

(i) $s_{n, k}$

\begin{tabular}{|cccccccccc|}
\hline 1 & 0 & 0 & 0 & 0 & 0 & 0 & 0 & 0 & 0 \\
1 & 1 & 0 & 0 & 0 & 0 & 0 & 0 & 0 & 0 \\
2 & 1 & 1 & 0 & 0 & 0 & 0 & 0 & 0 & 0 \\
$3-d$ & 2 & 1 & 1 & 0 & 0 & 0 & 0 & 0 & 0 \\
5 & 3 & 2 & 1 & 1 & 0 & 0 & 0 & 0 & 0 \\
7 & 5 & 3 & 2 & 1 & 1 & 0 & 0 & 0 & 0 \\
$-d^{2}-d+11$ & $7-d$ & 5 & 3 & 2 & 1 & 1 & 0 & 0 & 0 \\
15 & 11 & 7 & 5 & 3 & 2 & 1 & 1 & 0 & 0 \\
22 & 15 & 11 & 7 & 5 & 3 & 2 & 1 & 1 & 0 \\
$-d^{3}-2 d+30$ & $22-d$ & $15-d$ & 11 & 7 & 5 & 3 & 2 & 1 & 1 \\
\hline
\end{tabular}

(ii) $s_{n, k}^{(-1)}$

\begin{tabular}{|cccccccccc|}
\hline 1 & 0 & 0 & 0 & 0 & 0 & 0 & 0 & 0 & 0 \\
0 & 1 & 0 & 0 & 0 & 0 & 0 & 0 & 0 & 0 \\
0 & 1 & 1 & 0 & 0 & 0 & 0 & 0 & 0 & 0 \\
$-d$ & 1 & 1 & 1 & 0 & 0 & 0 & 0 & 0 & 0 \\
0 & 3 & 2 & 1 & 1 & 0 & 0 & 0 & 0 & 0 \\
0 & 2 & 2 & 2 & 1 & 1 & 0 & 0 & 0 & 0 \\
$-d^{2}-d$ & $7-d$ & 5 & 3 & 2 & 1 & 1 & 0 & 0 & 0 \\
$d$ & 7 & 6 & 4 & 3 & 2 & 1 & 1 & 0 & 0 \\
0 & 13 & 9 & 7 & 5 & 3 & 2 & 1 & 1 & 0 \\
$-d^{3}-2 d$ & $14-d$ & $13-d$ & 10 & 6 & 5 & 3 & 2 & 1 & 1 \\
\hline
\end{tabular}

(iii) $\gamma_{k}(n)$

Figure 2.2. A generalized factorization for $L_{a}(1,0,2,1 ; d, q)$

is that for $\alpha=\gamma$ and $\beta=\delta$ as in the generalized expansion of (1) from the introduction, the square matrix, $A_{n}:=\left(s_{i, j}\right)_{1<i, j<n}$, tends to not be invertible for $\alpha>1$. The statement in Theorem 2.5 does however allow us to formulate generalized analogs to the results in the references for some special cases of the parameters in (i) of the theorem. For example, if we consider the factorizations of the Lambert series expansions of the form

$$
L_{a}(1,0,2,1 ; q)=\sum_{n \geq 1} \frac{a_{n} q^{n}}{1-q^{2 n+1}},
$$

with $C(q):=(q ; q)_{\infty}$, we obtain non-singular matrices, $A_{n}$, as we have come to expect in the results from $[4,5]$ whose properties are summarized by Figure 2.1 where we define the corresponding inverse matrix entries to be of the form

$$
s_{n, k}^{(-1)}:=\sum_{d \mid n} p(d-k) \cdot \gamma_{k}(n / d),
$$

for some fixed sequence of arithmetic functions $\gamma_{k}$. We notice by experimentation with the integer sequences database [9] that for $k>\gamma+\delta$ in the expansion of $L(1,0, \gamma, \delta ; q)$ we appear to have that $\gamma_{k}(n)$ is related to partition functions with 
special generating functions of some underspecified sort. Experimentally, we also conjecture that ${ }^{1}$

$$
\begin{aligned}
s_{n, k}^{(-1)}=p(n-k)- & \sum_{i=1}^{n} p\left(\frac{n-i}{2 i+1}-k\right)[n \equiv i \bmod 2 i+1]_{\delta} \\
+ & +\sum_{m=2}^{n} \sum_{i=1}^{n} p\left(\frac{n-p(m+1) i-p(m-1)}{p(m+1)(2 i+1)}-k\right) \times \\
& \times[n \equiv p(m+1) i+p(m-1) \bmod p(m+1)(2 i+1)]_{\delta} .
\end{aligned}
$$

We can similarly define the analogous related expansions for the class of generalized Lambert series of the form

$$
L_{a}(1,0,2,1 ; d, q)=\sum_{n \geq 1} \frac{a_{n} q^{n}}{1-d \cdot q^{2 n+1}},
$$

so that the corresponding matrices of $s_{n, k}$ are also invertible. Figure 2.2 summarizes the characteristic expansions of the factorization when $C(q):=(q ; q)_{\infty}$. From our computational experimentation with the form of this series, we see that each of the entries in the tables listed in the figure are polynomials in $d$. Moreover, as in the first degenerate series case, we conjecture a related result that

$$
\begin{aligned}
s_{n, k}^{(-1)}=p(n-k) & -\sum_{i=1}^{n} d^{i} \cdot p\left(\frac{n-i}{2 i+1}-k\right)[n \equiv i \bmod 2 i+1]_{\delta} \\
& +\sum_{m=2}^{n} \sum_{i=1}^{n} d^{i} \cdot p\left(\frac{n-p(m+1) i-p(m-1)}{p(m+1)(2 i+1)}-k\right) \times \\
& \quad \times[n \equiv p(m+1) i+p(m-1) \bmod p(m+1)(2 i+1)]_{\delta} \\
& +p_{n, k}(d),
\end{aligned}
$$

where each $p_{n, k}(d)$ is a small polynomial in $d$ and is only non-zero in the rows $n$ indexed by the sequence $\{13,22,31,37,40,49,52,58,62,67,73, \ldots\}$.

We can generalized the result for the first degenerate case in (5) somewhat to state that if

$$
L_{a}(1,0, \alpha, 1 ; q)=\sum_{n \geq 1} \frac{a_{n} q^{n}}{1-q^{\alpha n+1}}=\frac{1}{C(q)} \sum_{n \geq 0} \sum_{k=1}^{n} s_{n, k}(\alpha, 1) a_{k} \cdot q^{n},
$$

for some $\alpha \geq 2$, then we have that

$$
s_{n, k}^{(-1)}(\alpha, 1):=p(n-k)-\sum_{i=1}^{n} p\left(\frac{n-i}{\alpha i+1}-k\right)[n \equiv i \bmod \alpha i+1]_{\delta}+p_{n, k}(\alpha, 1),
$$

where the sequence of $p_{n, k}(\alpha, 1)$ is integer-valued, mostly zero, and for the rows $n$ where $p_{n, k}(\alpha, 1)$ is non-zero this function is only non-zero for small cases of the columns $k$ with $k \ll n$. For example, when $\alpha:=3$ we have that the functions $p_{n, k}(3,1)$ are non-zero only for the small column cases in the rows $n \in$ $\{21,37,53,65,69,85,93,101,117,121,133,149, \ldots\}$, when $\alpha:=4$ the functions $p_{n, k}(4,1)$ are non-zero only for the rows $n \in\{31,56,81,101,106,131,146, \ldots\}$, and when $\alpha:=$

\footnotetext{
${ }^{1} \underline{\text { Notation: }}$ Iverson's convention compactly specifies boolean-valued conditions and is equivalent to the Kronecker delta function, $\delta_{i, j}$, as $[n=k]_{\delta} \equiv \delta_{n, k}$. Similarly, [cond $=$ True $]_{\delta} \equiv \delta_{\text {cond,True }}$ in the remainder of the article.
} 
5 the functions $p_{n, k}(5,1)$ are non-zero only for the rows $n \in\{43,79,115,145, \ldots\}$. The in-order non-zero indexed rows appear to have the same values for all $\alpha \geq 3$, i.e., $p_{21, k}(3,1)=p_{31, k}(4,1)=p_{43, k}(5,1)$, and so on.

\section{Variants of the Generalized Factorization Theorems}

The results in this section avoid the typically singular behavior of the matrices corresponding to the factorizations of (1) by construction and redefining the sequence $\bar{a}_{n}$ implicit to the more general factorization statement in (3). The next theorem provides the generalized analog to the variant of the ordinary factorization theorems proved in $[5, \S 3]$.

Theorem 3.1 (Another Generalized Factorization Theorem). If we define the factorization pair $\left(C(q), s_{n, k}\right)$ in (3) implicitly through (4) by

$$
s_{n, k}^{(-1)}:=\sum_{d \mid n}\left[q^{d-k}\right] \frac{1}{C(q)} \cdot \gamma(n / d),
$$

for some fixed arithmetic functions $\gamma(n)$ and $\widetilde{\gamma}(n):=\sum_{d \mid n} \gamma(d)$, then we have that the sequence of $\bar{a}_{n}$ is given by the following formula for all $n \geq 1$ :

$$
\bar{a}_{n}=\sum_{\substack{d \mid n \\ d \equiv \beta \bmod \alpha}} a_{\frac{d-\beta}{\alpha}} \widetilde{\gamma}(n / d) .
$$

Proof. The proof of this result is similar to the proof given in [5, Thm. 3.3]. In particular, we begin by noticing that

$$
\bar{a}_{n}=\sum_{k=1}^{n} s_{n, k}^{(-1)}\left[q^{k}\right]\left(\sum_{d=1}^{k} \frac{a_{d} q^{\alpha d+\beta}}{1-q^{\alpha d+\beta}}\right) .
$$

Then if we let $c_{n}:=\left[q^{n}\right] 1 / C(q)$ and let

$$
t_{k, d}(\alpha, \beta):=\left[q^{k}\right] \frac{q^{\alpha d+\beta}}{1-q^{\alpha d+\beta}} C(q),
$$

where $t_{i, d}(\alpha, \beta)=0$ whenever $i<\alpha d+\beta$, we have that for each $1 \leq d \leq n$

$$
\begin{aligned}
{\left[a_{d}\right] \bar{a}_{n} } & =\sum_{k=1}^{n} s_{n, k}^{(-1)} t_{k, d}(\alpha, \beta) \\
& =\sum_{k=d}^{n}\left(\sum_{r \mid n} c_{r-k} \gamma(n / r)\right) t_{k, d}(\alpha, \beta) \\
& =\sum_{r \mid n}\left(\sum_{i=d}^{r} c_{r-i} t_{i, d}(\alpha, \beta)\right) \gamma(n / r) \\
& =\sum_{r \mid n}\left(\sum_{i=0}^{r} c_{r-i} t_{i, d}(\alpha, \beta)\right) \gamma(n / r),
\end{aligned}
$$

where the inner sum is generated by

$$
\left[q^{r}\right] \frac{1}{C(q)} \frac{q^{\alpha d+\beta}}{1-q^{\alpha d+\beta}} C(q)=[\alpha d+\beta \mid r]_{\delta} .
$$


Thus we have that

$$
\left[a_{d}\right] \bar{a}_{n}=\sum_{\substack{r|n \\ \alpha d+\beta| r}} \gamma(n / d),
$$

and then that

$$
\begin{aligned}
\bar{a}_{n} & =\sum_{\substack{d \mid n \\
d \equiv \beta \bmod \alpha}} a_{\frac{d-\beta}{\alpha}} \sum_{\substack{r|n \\
d| r}} \gamma(n / r) \\
& =\sum_{\substack{d \mid n \\
d \equiv \beta \bmod \alpha}} a_{\frac{d-\beta}{\alpha}} \sum_{r \mid \frac{n}{d}} \gamma\left(\frac{n}{d r}\right) .
\end{aligned}
$$

We choose to phrase the next few results cited in Example 3.2 in terms of the special case function $C(q) \equiv(q ; q)_{\infty}$ whose reciprocal generates Euler's partition function as $p(n):=\left[q^{n}\right](q ; q)_{\infty}^{-1}$ in the first form of the factorization assumed by Theorem 3.1. We note that these identities could just as well be expanded in terms of other reciprocal generating functions for special partition functions are defined through the choice of $C(q)$, such as the partition function $q(n)$ corresponding to $C(q) \equiv(-q ; q)_{\infty}^{-1}$.

Example 3.2 (Applications of the Theorem). We begin by noticing that for a fixed function $C(q)$, the general expansions of the factorization result stated in (3) implies that we have

$$
\bar{a}_{n}=\sum_{k=1}^{n} s_{n, k}^{(-1)} B_{k-1},
$$

where $B_{k}$ depends implicitly only on the selection of $a_{n}$ according to the next sums over the paired function $b_{m}$ in (1) for $k \geq 1$ :

$$
\begin{aligned}
B_{k-1} & =\sum_{k=1}^{n} s_{n, k} a_{k} \\
& =b_{k}+\sum_{k=1}^{n}\left[q^{n}\right] \frac{1}{C(q)} \cdot b_{n-k} \\
& =\sum_{\alpha d-\beta \mid n} a_{d}+\sum_{k=1}^{n}\left[q^{n}\right] \frac{1}{C(q)} \cdot\left(\sum_{\alpha d-\beta \mid n-k} a_{d}\right) .
\end{aligned}
$$

For example, when we take $\left(a_{n}, \gamma(n)\right):=\left(1, n^{t}\right)$ for some $t \in \mathbb{C}$ in Theorem 3.1, we obtain the result

$$
\begin{aligned}
& \sum_{\substack{d \mid n \\
d \equiv}} \sigma_{t}\left(\frac{n}{d}\right) \\
= & \sum_{k=1}^{n}\left(\sum_{d \mid n} p(d-k)(n / d)^{t}\right)\left[b_{k}(1 ; \alpha, \beta)+\sum_{s= \pm 1} \sum_{j=1}^{\left\lfloor\frac{\sqrt{24 k+1}-s}{6}\right\rfloor} b_{k-\frac{j(3 j+s)}{2}}(1 ; \alpha, \beta)\right],
\end{aligned}
$$


where we define the notation for $b_{k}(1 ; \alpha, \beta)$ to be the special case of the restricted divisor sums over $a_{n}$ given by

$$
b_{k}\left(a_{n} ; \alpha, \beta\right):=\sum_{\alpha d-\beta \mid k} a_{d} .
$$

If we next define $\left(a_{n}, \gamma(n)\right):=\left(n^{s}, n^{t}\right),\left(n^{s}, \phi(n)\right)$, respectively, we similarly obtain the following identities as corollaries of the theorem above:

$$
\begin{aligned}
& \sum_{\substack{d \mid n \\
d \equiv \beta \bmod \alpha}}\left(\frac{d-\beta}{\alpha}\right)^{s} \cdot \sigma_{t}(n / d) \\
= & \sum_{k=1}^{n}\left(\sum_{d \mid n} p(d-k)(n / d)^{t}\right)\left[b_{k}\left(n^{s} ; \alpha, \beta\right)+\sum_{s= \pm 1} \frac{\left\lfloor\frac{\sqrt{24 k+1-s}\rfloor}{6}\right\rfloor}{\sum_{j=1}^{t}} b_{k-\frac{j(3 j+s)}{2}}\left(n^{s} ; \alpha, \beta\right)\right] \\
n \times & \sum_{d \mid n} \frac{1}{d} \cdot\left(\frac{d-\beta}{\alpha}\right)^{t} \\
= & \sum_{k=1}^{n}\left(\sum_{d \mid n} p(d-k) \phi(n / d)\right)\left[b_{k}\left(n^{t} ; \alpha, \beta\right)+\sum_{s= \pm 1} \frac{\left\lfloor\frac{\sqrt{24 k+1-s}}{\sum_{j=1}^{6}}\right\rfloor}{b_{k-\frac{j(3 j+s)}{2}}}\left(n^{t} ; \alpha, \beta\right)\right] .
\end{aligned}
$$

The generalized expansions of the well-known classical Lambert series stated in (2) also imply the next corollaries of the theorem for some fixed $s, t \in \mathbb{C}$.

$$
\begin{aligned}
& \sum_{\substack{d \mid n \\
d \equiv \beta \bmod \alpha}} \log (d) \\
= & \sum_{k=1}^{n}\left(\sum_{d \mid n} p(d-k) \Lambda(n / d)\right)\left[b_{k}(1 ; \alpha, \beta)+\sum_{s= \pm 1} \sum_{j=1}^{\left\lfloor\frac{\sqrt{24 k+1}-s}{6}\right\rfloor} b_{k-\frac{j(3 j+s)}{2}}(1 ; \alpha, \beta)\right] \\
& \sum_{\substack{d \mid n \\
d \equiv \bmod \alpha}}\left(\frac{d-\beta}{\alpha}\right)^{s} \cdot\left(\frac{n}{d}\right)^{t} \\
= & \left.\sum_{k=1}^{n}\left(\sum_{d \mid n} p(d-k) J_{t}(n / d)\right)\left[b_{k}\left(n^{s} ; \alpha, \beta\right)+\sum_{s= \pm 1} \frac{\left.\frac{\sqrt{24 k+1-s}}{6}\right\rfloor}{\sum_{j=1}^{6}} b_{k-\frac{j(3 j+s)}{2}}\left(n^{s} ; \alpha, \beta\right)\right)\right] .
\end{aligned}
$$

We also have expansions of related identities involving the sum of squares function, $r_{2}(n)=\left[q^{n}\right] \vartheta_{3}(q)^{2}$, in the special case where $\left(\alpha, \beta, a_{n}\right):=\left(2,1,4 \cdot(-1)^{n+1}\right)$. In particular, for any prescribed arithmetic functions $a_{n}$ and $\gamma(n)$ such that $\widetilde{\gamma}(n):=$ $\sum_{d \mid n} \gamma(d)$, we have expansions involving $r_{2}(n)$ given by

$$
\sum_{\substack{d \mid n \\ d \text { odd }}} a_{\frac{d-1}{2}} r_{2}(n / d)=\sum_{k=1}^{n} \sum_{d \mid n} 4 \cdot(-1)^{n / d+1} p(d-k)\left[b_{k}\left(a_{n} ; 2,1\right)\right.
$$




$$
\left.+\sum_{s= \pm 1} \sum_{j=1}^{\left\lfloor\frac{\sqrt{24 k+1}-s}{6}\right\rfloor} b_{k-\frac{j(3 j+s)}{2}}\left(a_{n} ; 2,1\right)\right]
$$

and expanded by

$$
\begin{gathered}
\sum_{\substack{d \mid n \\
d \text { odd }}} 4 \cdot(-1)^{(d+1) / 2} \widetilde{\gamma}(n / d) \\
=\sum_{k=1}^{n} \sum_{d \mid n} p(d-k) \gamma(n / d) \cdot\left[q^{k}\right](q ; q)_{\infty} \vartheta_{3}(q)^{2} \\
\sum_{\substack{d \mid n \\
d \text { odd }}}(-1)^{(d+1) / 2}\left(r_{2}\left(\frac{n}{d}\right)-4 \cdot d\left(\frac{n}{2 d}\right)\left[\frac{n}{d} \text { even }\right]_{\delta}\right) \\
\quad=\sum_{k=1}^{n} \sum_{d \mid n} p(d-k)(-1)^{n / d+1} \cdot\left[q^{k}\right](q ; q)_{\infty} \vartheta_{3}(q)^{2},
\end{gathered}
$$

where $d(n) \equiv \sigma_{0}(n)$ denotes the divisor function and where the powers of the Jacobi theta function, $\vartheta_{3}(q):=1+2 \sum_{n \geq 1} q^{n^{2}}$, generate the more general sums of the sums of $k$ squares functions, $r_{k}(n):=\left[q^{n}\right] \vartheta_{3}(q)^{k}$.

\section{FACTORIZAtion theOREMS FOR LAMBERT SERIES OVER CONVOLUtions OF ARITHMETIC FUNCTIONS}

4.1. Overview and definitions. Given two prescribed arithmetic functions $f$ and $g$ we define their convolution, or Dirichlet convolution, denoted by $h=f * g$, to be the function

$$
(f * g)(n):=\sum_{d \mid n} f(d) g(n / d),
$$

for all natural numbers $n \geq 1[1, \S 2.6]$. The usual Möbius inversion result is stated in terms of convolutions as follows where $\mu$ is the Möbius function: $h=f * 1$ if and only if $f=h * \mu$. There is a natural connection between the coefficients of the Lambert series of an arithmetic function $a_{n}$ and its corresponding Dirichlet generating function, $\operatorname{DGF}\left(a_{n} ; s\right):=\sum_{n \geq 1} a_{n} / n^{s}$. Namely, we have that for any $s \in \mathbb{C}$ such that $\Re(s)>1$

$$
b_{n}=\left[q^{n}\right] \sum_{n \geq 1} \frac{a_{n} q^{n}}{1-q^{n}} \quad \text { if and only if } \quad \operatorname{DGF}\left(b_{n} ; s\right)=\operatorname{DGF}\left(a_{n} ; s\right) \zeta(s),
$$

where $\zeta(s)$ is the Riemann zeta function. Moreover, we can further connect the coefficients of the Lambert series over a convolution of arithmetic functions to its associated Dirichlet series by noting that $\operatorname{DGF}(f * g ; s)=\operatorname{DGF}(f ; s) \cdot \operatorname{DGF}(g ; s)$.

In this section, we consider the generalized Lambert series factorization theorems in the context of (1) where the function $a_{n}$ is defined to be a convolution of two arithmetic functions. Since we have not yet explored factorization theorems of this type in the references $(c f .[4,5])$, we first state a few factorization results for Lambert series over these convolution functions in the "ordinary" case of $(1)$ where $(\alpha, \beta):=$ $(1,0)$. More precisely, we give the statements and proofs of Proposition 4.1 and a 
closely-related theorem next and then proceed to evaluate several consequences and special case formulations following from these results below.

\begin{tabular}{|c|c|c|c|c|c|c|c|c|c|c|c|c|c|c|c|c|c|c|c|c|}
\hline 1 & 0 & 0 & 0 & 0 & 0 & 0 & 0 & 0 & 0 & 0 & 0 & 0 & 0 & 0 & 0 & 0 & 0 & 0 & 0 & 0 \\
\hline 1 & 1 & 0 & 0 & 0 & 0 & 0 & 0 & 0 & 0 & 0 & 0 & 0 & 0 & 0 & 0 & 0 & 0 & 0 & 0 & 0 \\
\hline 1 & 1 & 0 & 0 & 0 & 0 & 0 & 0 & 0 & 0 & 0 & 0 & 0 & 0 & 0 & 0 & 0 & 0 & 0 & 0 & 0 \\
\hline 1 & 2 & 1 & 0 & 0 & 0 & 0 & 0 & 0 & 0 & 0 & 0 & 0 & 0 & 0 & 0 & 0 & 0 & 0 & 0 & 0 \\
\hline 1 & 1 & 0 & 0 & 0 & 0 & 0 & 0 & 0 & 0 & 0 & 0 & 0 & 0 & 0 & 0 & 0 & 0 & 0 & 0 & 0 \\
\hline 1 & 3 & 2 & 0 & 0 & 0 & 0 & 0 & 0 & 0 & 0 & 0 & 0 & 0 & 0 & 0 & 0 & 0 & 0 & 0 & 0 \\
\hline 1 & 1 & 0 & 0 & 0 & 0 & 0 & 0 & 0 & 0 & 0 & 0 & 0 & 0 & 0 & 0 & 0 & 0 & 0 & 0 & 0 \\
\hline 1 & 3 & 3 & 1 & 0 & 0 & 0 & 0 & 0 & 0 & 0 & 0 & 0 & 0 & 0 & 0 & 0 & 0 & 0 & 0 & 0 \\
\hline 1 & 2 & 1 & 0 & 0 & 0 & 0 & 0 & 0 & 0 & 0 & 0 & 0 & 0 & 0 & 0 & 0 & 0 & 0 & 0 & 0 \\
\hline 1 & 3 & 2 & 0 & 0 & 0 & 0 & 0 & 0 & 0 & 0 & 0 & 0 & 0 & 0 & 0 & 0 & 0 & 0 & 0 & 0 \\
\hline 1 & 1 & 0 & 0 & 0 & 0 & 0 & 0 & 0 & 0 & 0 & 0 & 0 & 0 & 0 & 0 & 0 & 0 & 0 & 0 & 0 \\
\hline 1 & 5 & 7 & 3 & 0 & 0 & 0 & 0 & 0 & 0 & 0 & 0 & 0 & 0 & 0 & 0 & 0 & 0 & 0 & 0 & 0 \\
\hline 1 & 1 & 0 & 0 & 0 & 0 & 0 & 0 & 0 & 0 & 0 & 0 & 0 & 0 & 0 & 0 & 0 & 0 & 0 & 0 & 0 \\
\hline 1 & 3 & 2 & 0 & 0 & 0 & 0 & 0 & 0 & 0 & 0 & 0 & 0 & 0 & 0 & 0 & 0 & 0 & 0 & 0 & 0 \\
\hline 1 & 3 & 2 & 0 & 0 & 0 & 0 & 0 & 0 & 0 & 0 & 0 & 0 & 0 & 0 & 0 & 0 & 0 & 0 & 0 & 0 \\
\hline 1 & 4 & 6 & 4 & 1 & 0 & 0 & 0 & 0 & 0 & 0 & 0 & 0 & 0 & 0 & 0 & 0 & 0 & 0 & 0 & 0 \\
\hline 1 & 1 & 0 & 0 & 0 & 0 & 0 & 0 & 0 & 0 & 0 & 0 & 0 & 0 & 0 & 0 & 0 & 0 & 0 & 0 & 0 \\
\hline 1 & 5 & 7 & 3 & 0 & 0 & 0 & 0 & 0 & 0 & 0 & 0 & 0 & 0 & 0 & 0 & 0 & 0 & 0 & 0 & 0 \\
\hline 1 & 1 & 0 & 0 & 0 & 0 & 0 & 0 & 0 & 0 & 0 & 0 & 0 & 0 & 0 & 0 & 0 & 0 & 0 & 0 & 0 \\
\hline 1 & 5 & 7 & 3 & 0 & 0 & 0 & 0 & 0 & 0 & 0 & 0 & 0 & 0 & 0 & 0 & 0 & 0 & 0 & 0 & 0 \\
\hline 1 & 3 & 2 & 0 & 0 & 0 & 0 & 0 & 0 & 0 & 0 & 0 & 0 & 0 & 0 & 0 & 0 & 0 & 0 & 0 & 0 \\
\hline 1 & 3 & 2 & 0 & 0 & 0 & 0 & 0 & 0 & 0 & 0 & 0 & 0 & 0 & 0 & 0 & 0 & 0 & 0 & 0 & 0 \\
\hline 1 & 1 & 0 & 0 & 0 & 0 & 0 & 0 & 0 & 0 & 0 & 0 & 0 & 0 & 0 & 0 & 0 & 0 & 0 & 0 & 0 \\
\hline 1 & 7 & 15 & 13 & 4 & 0 & 0 & 0 & 0 & 0 & 0 & 0 & 0 & 0 & 0 & 0 & 0 & 0 & 0 & 0 & 0 \\
\hline 1 & 2 & 1 & 0 & 0 & 0 & 0 & 0 & 0 & 0 & 0 & 0 & 0 & 0 & 0 & 0 & 0 & 0 & 0 & 0 & 0 \\
\hline 1 & 3 & 2 & 0 & 0 & 0 & 0 & 0 & 0 & 0 & 0 & 0 & 0 & 0 & 0 & 0 & 0 & 0 & 0 & 0 & 0 \\
\hline 1 & 3 & 3 & 1 & 0 & 0 & 0 & 0 & 0 & 0 & 0 & 0 & 0 & 0 & 0 & 0 & 0 & 0 & 0 & 0 & 0 \\
\hline 1 & 5 & 7 & 3 & 0 & 0 & 0 & 0 & 0 & 0 & 0 & 0 & 0 & 0 & 0 & 0 & 0 & 0 & 0 & 0 & 0 \\
\hline 1 & 1 & 0 & 0 & 0 & 0 & 0 & 0 & 0 & 0 & 0 & 0 & 0 & 0 & 0 & 0 & 0 & 0 & 0 & 0 & 0 \\
\hline 1 & 7 & 12 & 6 & 0 & 0 & 0 & 0 & 0 & 0 & 0 & 0 & 0 & 0 & 0 & 0 & 0 & 0 & 0 & 0 & 0 \\
\hline 1 & 1 & 0 & 0 & 0 & 0 & 0 & 0 & 0 & 0 & 0 & 0 & 0 & 0 & 0 & 0 & 0 & 0 & 0 & 0 & 0 \\
\hline 1 & 5 & 10 & 10 & 5 & 1 & 0 & 0 & 0 & 0 & 0 & 0 & 0 & 0 & 0 & 0 & 0 & 0 & 0 & 0 & 0 \\
\hline 1 & 3 & 2 & 0 & 0 & 0 & 0 & 0 & 0 & 0 & 0 & 0 & 0 & 0 & 0 & 0 & 0 & 0 & 0 & 0 & 0 \\
\hline 1 & 3 & 2 & 0 & 0 & 0 & 0 & 0 & 0 & 0 & 0 & 0 & 0 & 0 & 0 & 0 & 0 & 0 & 0 & 0 & 0 \\
\hline 1 & 3 & 2 & 0 & 0 & 0 & 0 & 0 & 0 & 0 & 0 & 0 & 0 & 0 & 0 & 0 & 0 & 0 & 0 & 0 & 0 \\
\hline 1 & 8 & 19 & 18 & 6 & 0 & 0 & 0 & 0 & 0 & 0 & 0 & 0 & 0 & 0 & 0 & 0 & 0 & 0 & 0 & 0 \\
\hline 1 & 1 & 0 & 0 & 0 & 0 & 0 & 0 & 0 & 0 & 0 & 0 & 0 & 0 & 0 & 0 & 0 & 0 & 0 & 0 & 0 \\
\hline 1 & 3 & 2 & 0 & 0 & 0 & 0 & 0 & 0 & 0 & 0 & 0 & 0 & 0 & 0 & 0 & 0 & 0 & 0 & 0 & 0 \\
\hline 1 & 3 & 2 & 0 & 0 & 0 & 0 & 0 & 0 & 0 & 0 & 0 & 0 & 0 & 0 & 0 & 0 & 0 & 0 & 0 & 0 \\
\hline 1 & 7 & 15 & 13 & 4 & 0 & 0 & 0 & 0 & 0 & 0 & 0 & 0 & 0 & 0 & 0 & 0 & 0 & 0 & 0 & 0 \\
\hline 1 & 1 & 0 & 0 & 0 & 0 & 0 & 0 & 0 & 0 & 0 & 0 & 0 & 0 & 0 & 0 & 0 & 0 & 0 & 0 & 0 \\
\hline 1 & 7 & 12 & 6 & 0 & 0 & 0 & 0 & 0 & 0 & 0 & 0 & 0 & 0 & 0 & 0 & 0 & 0 & 0 & 0 & 0 \\
\hline 1 & 1 & 0 & 0 & 0 & 0 & 0 & 0 & 0 & 0 & 0 & 0 & 0 & 0 & 0 & 0 & 0 & 0 & 0 & 0 & 0 \\
\hline 1 & 5 & 7 & 3 & 0 & 0 & 0 & 0 & 0 & 0 & 0 & 0 & 0 & 0 & 0 & 0 & 0 & 0 & 0 & 0 & 0 \\
\hline 1 & 5 & 7 & 3 & 0 & 0 & 0 & 0 & 0 & 0 & 0 & 0 & 0 & 0 & 0 & 0 & 0 & 0 & 0 & 0 & 0 \\
\hline 1 & 3 & 2 & 0 & 0 & 0 & 0 & 0 & 0 & 0 & 0 & 0 & 0 & 0 & 0 & 0 & 0 & 0 & 0 & 0 & 0 \\
\hline 1 & 1 & 0 & 0 & 0 & 0 & 0 & 0 & 0 & 0 & 0 & 0 & 0 & 0 & 0 & 0 & 0 & 0 & 0 & 0 & 0 \\
\hline 1 & 9 & 26 & 34 & 21 & 5 & 0 & 0 & 0 & 0 & 0 & 0 & 0 & 0 & 0 & 0 & 0 & 0 & 0 & 0 & 0 \\
\hline 1 & 2 & 1 & 0 & 0 & 0 & 0 & 0 & 0 & 0 & 0 & 0 & 0 & 0 & 0 & 0 & 0 & 0 & 0 & 0 & 0 \\
\hline 1 & 5 & 7 & 3 & 0 & 0 & 0 & 0 & 0 & 0 & 0 & 0 & 0 & 0 & 0 & 0 & 0 & 0 & 0 & 0 & 0 \\
\hline
\end{tabular}

Table 4.1. The functions $\mathrm{ds}_{j, 1}(n)$ for columns $1 \leq j \leq 21$ and $1 \leq n \leq 50$

\subsection{Main results.}

Proposition 4.1 (One Possible Factorization). Let $f$ and $g$ denote non-identicallyzero arithmetic functions. Suppose that we have an ordinary Lambert series factorization for any prescribed arithmetic function $a_{n}$ of the form

$$
\sum_{n \geq 1} \frac{a_{n} q^{n}}{1-q^{n}}=\frac{1}{C(q)} \sum_{n \geq 1} \sum_{k=1}^{n} s_{n, k} a_{k} \cdot q^{n},
$$

so that, for example, when $C(q):=(q ; q)_{\infty}$ we have that $s_{n, k}=s_{o}(n, k)-s_{e}(n, k)$ where $s_{o}(n, k)$ and $s_{e}(n, k)$ respectively denote the number of $k$ 's in all partitions of 
$n$ into an odd (even) number of distinct parts. Then we have one possible formulation of a factorization theorem for the Lambert series over the convolution function $h=f * g$ expanded as

$$
\sum_{n \geq 1} \frac{(f * g)(n) q^{n}}{1-q^{n}}=\frac{1}{C(q)} \sum_{n \geq 1} \sum_{k=1}^{n} \widetilde{s}_{n, k}(g) f(k) \cdot q^{n},
$$

where

$$
\widetilde{s}_{n, k}(g)=\sum_{j=1}^{n} s_{n, k j} \cdot g(j) .
$$

Proof. It is apparent by the expansions on the left-hand-side of (ii) that there is some sequence of $\widetilde{s}_{n, k}(g)$ depending on the function $g$ that satisfies the factorization of the form in (i) when $a_{n} \mapsto(f * g)(n)$. For a fixed $k \geq 1$, we begin by evaluating the coefficients of $f(k)$ on the right-hand-side of (ii) as follows:

$$
\begin{aligned}
{[f(k)]\left(\sum_{n \geq 1} \sum_{i=1}^{n} s_{n, i}(f * g)(i) \cdot q^{n}\right) } & =\sum_{n \geq 1} \sum_{i=1}^{n}[f(k)] s_{n, i} \sum_{d \mid i} f(d) g(i / d) \cdot q^{n} \\
& =\sum_{n \geq 1} \sum_{i=1}^{n} s_{n, i} g(i / k)[k \mid i]_{\delta} \cdot q^{n} \\
& =\sum_{n \geq 1}\left(\sum_{j=1}^{n} s_{n, k j} \cdot g(j)\right) q^{n} .
\end{aligned}
$$

Thus we have that the formula for $\widetilde{s}_{n, k}(g)$ given in (iii) is correct as claimed.

By Corollary 2.6 we can easily generalize this result to the analogous series expansions of (1). However, in these cases the matrix of $s_{n, k}$ is typically singular so that we are unable to formulate an analog to the following theorem for the inverse sequences which implies many useful and interesting new results discussed in the following examples.

Notation: For a fixed arithmetic function $g$ with $g(1):=1$, let the functions $\mathrm{ds}_{j, g}(n)$ be defined recursively for natural numbers $j \geq 1$ as

$$
\mathrm{ds}_{j, g}(n):= \begin{cases}g_{ \pm}(n), & \text { if } j=1 ; \\ \sum_{d \mid n} g(d) \mathrm{ds}_{j-1, g}(n / d), & \text { if } j>1, \\ d>1 & \end{cases}
$$

where $g_{ \pm}(n):=g(n)[n>1]_{\delta}-\delta_{n, 1}$ and let the notation for the $k$-shifted partition function be defined as $p_{k}(n):=p(n-k)$ for $k \geq 1$. If we let the function $\widetilde{\mathrm{ds}}_{j, g}(n)$ denote the $j$-fold convolution of $g$ with itself, i.e., that

$$
\widetilde{\mathrm{ds}}_{j, g}(n)=\underbrace{\left(g_{ \pm} * g * \cdots * g\right)}_{n \text { times }}(n),
$$

then we can prove easily by induction that for all $m, n \geq 1$ we have the expansion

$$
\mathrm{ds}_{m, g}(n)=\sum_{i=0}^{m-1}\left(\begin{array}{c}
m-1 \\
i
\end{array}\right)(-1)^{m-1-i} \cdot \widetilde{\mathrm{ds}}_{i+1, g}(n) .
$$


We then define the following notation for the sums of the variant convolution functions for use in the theorem below when $n \geq 1$ :

$$
D_{n, g}(n):=\sum_{j=1}^{n} \mathrm{ds}_{2 j, g}(n)=\sum_{m=1}^{\lfloor n / 2\rfloor} \sum_{i=0}^{2 m-1}\left(\begin{array}{c}
2 m-1 \\
i
\end{array}\right)(-1)^{i+1} \widetilde{\mathrm{ds}}_{i+1, g}(n) .
$$

A listing of the values of the functions $\mathrm{ds}_{j, g}(n)$ in the special form when $g(n) \equiv 1$ is tabulated in Table 4.1. The sum

$$
\sum_{i=1}^{n} \mathrm{ds}_{2 i, 1}(n) \mapsto\{0,1,1,2,1,3,1,4,2,3,1,8,1,3,3,8, \ldots\},
$$

and its Möbius transform appear in the integer sequences database as the number of perfect partitions of $n$ [9, A002033, A174726]. The next result provides the form of the inverse sequences, $s_{n, k}^{(-1)}$, in the expansions proved in the proposition immediately above.

\begin{tabular}{|cccccccccc|}
\hline 1 & 0 & 0 & 0 & 0 & 0 & 0 & 0 & 0 & 0 \\
0 & 1 & 0 & 0 & 0 & 0 & 0 & 0 & 0 & 0 \\
0 & -1 & 1 & 0 & 0 & 0 & 0 & 0 & 0 & 0 \\
0 & 0 & -1 & 1 & 0 & 0 & 0 & 0 & 0 & 0 \\
0 & -1 & -1 & -1 & 1 & 0 & 0 & 0 & 0 & 0 \\
0 & 1 & 1 & -1 & -1 & 1 & 0 & 0 & 0 & 0 \\
0 & -1 & -1 & 0 & -1 & -1 & 1 & 0 & 0 & 0 \\
0 & 1 & 0 & 1 & 0 & -1 & -1 & 1 & 0 & 0 \\
0 & -1 & 2 & 0 & 0 & 0 & -1 & -1 & 1 & 0 \\
0 & 2 & -1 & -1 & 2 & 0 & 0 & -1 & -1 & 1 \\
0 & -2 & -1 & 1 & -1 & 1 & 0 & 0 & -1 & -1 \\
0 & 2 & 3 & 2 & 0 & 1 & 1 & 0 & 0 & -1 \\
0 & -2 & -2 & -1 & 0 & 0 & 0 & 1 & 0 & 0 \\
0 & 3 & -1 & -2 & 0 & -1 & 2 & 0 & 1 & 0 \\
0 & -3 & 4 & 1 & 3 & 0 & -1 & 1 & 0 & 1 \\
0 & 3 & -3 & 2 & -2 & 0 & -1 & 1 & 1 & 0 \\
0 & -4 & -2 & -1 & -2 & 1 & 0 & -1 & 0 & 1 \\
0 & 5 & 5 & -3 & 0 & 1 & 0 & -1 & 1 & 0 \\
0 & -5 & -4 & 1 & 0 & -1 & 0 & 0 & -1 & 0 \\
0 & 5 & -2 & 4 & 4 & -2 & 0 & -1 & -1 & 1 \\
0 & -6 & 8 & -2 & -3 & -1 & 3 & 1 & -1 & -1 \\
\hline
\end{tabular}

Table 4.2. The sequences $\rho_{n, 1}^{(i)}$ for the first rows $1 \leq n \leq 21$ and columns $1 \leq i \leq 10$

Theorem 4.2 (Inverse Sequences). When $C(q):=(q ; q)_{\infty}$, the inverse sequences from Proposition 4.1 satisfy

$$
\begin{aligned}
\sum_{d \mid n} s_{n, k}^{(-1)}(g) & =p_{k}(n)+\sum_{j=1}^{\Omega(n)}\left(p_{k} * \mathrm{ds}_{2 j, g}\right)(n) \\
& =p_{k}(n)+\left(p_{k} * D_{n, g}\right)(n),
\end{aligned}
$$


which by Möbius inversion is equivalent to

$$
\begin{aligned}
s_{n, k}^{(-1)}(g) & =\left(p_{k} * \mu\right)(n)+\sum_{j=1}^{\Omega(n)}\left(p_{k} * \operatorname{ds}_{2 j, g} * \mu\right)(n) \\
& =\left(p_{k} * \mu\right)(n)+\left(p_{k} * D_{n, g} * \mu\right)(n),
\end{aligned}
$$

where $D_{n, g}(n)$ is defined as in the remarks given above. We note that strictly speaking replacing the upper bound on the first sum above with $n$ in place of $\Omega(n)$ has no effect on the value of the sum.

Proof of the Claim. Let the proposed inverse sequence function be defined in the following notation which we denote in shorthand by $\widehat{s}_{k} \equiv \widehat{s}_{k}(n)$ :

$$
\widehat{s}_{n, k}^{(-1)}(g):=\left(p_{k} * \mu\right)(n)+\left(p_{k} * D_{n, g} * \mu\right)(n) .
$$

We begin as in the proof of Theorem 3.2 in [4] and consider the ordinary, nonconvolved Lambert series over the function $\widehat{s}_{k}$. More precisely, by the expansion in (i) of the proposition we must show that

$$
\begin{aligned}
\sum_{d \mid n} \widehat{s}_{d, k}^{(-1)} & :=p_{k}(n)+\left(p_{k} * D_{n, g}\right)(n) \\
& =\sum_{m=0}^{n} \sum_{j=1}^{n-m} \widetilde{s}_{n-m, j}(g) \cdot \widehat{s}_{j, k}^{(-1)} \cdot p(m) .
\end{aligned}
$$

For $n, k, i \geq 1$ with $k, i \leq n$, let the coefficient functions, $\rho_{n, k}^{(i)}$ be defined as

$$
\rho_{n, k}^{(i)}:=\sum_{j=1}^{n} s_{n, i j} \cdot \widetilde{s}_{j, k}^{(-1)} .
$$

Then for any fixed arithmetic function $h$ we can prove (by considering the related expansions of the factorizations in (ii) of the proposition for $\widehat{s}_{k} * g$ ) that

$$
t_{n, k}(h):=\sum_{j=1}^{n} s_{n, j} \cdot\left(\widetilde{s}_{n, k}^{(-1)} * h\right)(j)=\sum_{i=1}^{n} \rho_{n, k}^{(i)} \cdot h(i) .
$$

It remains to show that

$$
\sum_{m=0}^{n} t_{n-m, k}(h) \cdot p(m)=\left(p_{k} * h\right)(n) .
$$

Since we can expand the left-hand-side of the previous sum as

$$
\sum_{m=0}^{n} \sum_{i=1}^{n-m} \rho_{n-m, k}^{(i)} \cdot h(i) \cdot p(m)=\sum_{i=1}^{n} h(i) \underbrace{\left(\sum_{m=0}^{n} \rho_{n-m, k}^{(i)} \cdot p(m)\right)}_{:=u_{n, k}^{(i)}},
$$

to complete the proof of (ii) we need to prove a subclaim that (I) $u_{n, k}^{(i)}=0$ if $i \not n$; and (II) if $i \mid n$ then $u_{n, k}^{(i)}=p\left(\frac{n}{i}-k\right)$.

Proof of Subclaim: For $i:=1$, this is clearly the case since $\rho_{n-m, k}^{(i)}=[n-m=k]_{\delta}$. For subsequent cases of $i \geq 2$, it is apparent that

$$
\rho_{n, k}^{(i)}=\rho_{n-(k-1) i, 1}
$$


much as in the cases of the tables for the inverse sequences, $s_{n, k}^{(-1)}=\left(p_{k} * \mu\right)(n)$, given in the reference $[4, \S 3]$ (see Table 4.2). Finally, we claim that generating functions for the sequences of $u_{n, k}^{(i)}$ for each $i \geq 2$ are expanded in the form of

$$
\sum_{n \geq 0} u_{n, k}^{(i)} \cdot q^{n}=\prod_{j=1}^{i-1}\left(q^{j} ; q^{i}\right)_{\infty} \times \frac{q^{i k}}{(q ; q)_{\infty}}=q^{i k} \cdot \sum_{n \geq 0} p(n) q^{i n}
$$

which we see by comparing coefficients on the right-hand-side of the previous equation implies our claim.

Completing the Proof of the Inverse Formula: What we have shown by proving (ii) above is an inverse formula for an ordinary Lambert series factorization over the sequence of $a_{j}:=\left(\widetilde{s}_{n, k}^{(-1)} * g\right)(j)$. In particular, by Möbius inversion (ii) shows that we have

$$
\begin{aligned}
(f * g)(n)=\left(s_{n, k}^{(-1)} * g\right)(n) & \Longleftrightarrow\left(f-s_{n, k}^{(-1)}\right) * g \equiv 0 \\
& \Longrightarrow f_{n}=s_{n, k}^{(-1)}, \text { when } g \equiv 0
\end{aligned}
$$

More to the point, when we define $f_{n}:=\widetilde{s}_{n, k}^{(-1)}(g)$ where by convenience and experimental suggestion we let

$$
\widetilde{s}_{n, k}^{(-1)}(g)=s_{n, k}^{(-1)} * t_{n, k}^{(-1)}(g),
$$

for some convolution-wise factorization of this inverse sequence, we can now prove the exact formula for the inverse sequence claimed in the theorem statement. In the forward direction, we suppose that

$$
t_{n, k}^{(-1)}(g)=D_{n, g}(n)+\varepsilon(n)
$$

where $\varepsilon(n)=\delta_{n, 1}$ is the multiplicative identity and then see from the formulas for $D_{n, g}(n)$ discussed before the claim that $g *\left(D_{n, g}+\varepsilon\right)=\varepsilon$, which proves that our inverse formula is correct in this case. Conversely, if we require that

$$
s_{n, k}^{(-1)} * t_{n, k}^{(-1)}(g) * g=s_{n, k}^{(-1)}
$$

for all $n$ and choices of the function $g$, we must have that $t_{n, k}^{(-1)} * g=\varepsilon$, and so we see that $t_{n, k}^{(-1)}=D_{n, g}+\varepsilon$ as required. That is to say, we have proved our result using the implicit statement that $t * g=\varepsilon$ if and only if $t=D_{n, g}+\varepsilon$, i.e., that $t=D_{n, g}+\varepsilon$ is the unique function such that $t * g=\varepsilon$ for all $n \geq 1$, a result which we do not prove here and only mention for the sake of brevity.

4.3. Corollaries and applications. We have several immediate consequences of the theorem, which in some respects follows naturally as a corollary of the expansions of the proposition above. Some of these applications are discussed in the next example.

Example 4.3 (Applications). To ease the notation in the applications that follow, for integers $n \geq 0$ we define the functions $B_{n}(f * g)$ in the shorthand of

$$
B_{n}(f * g):=\left[q^{n+1}\right]\left(\sum_{m \geq 1} \frac{(f * g)(n) q^{n} \times(q ; q)_{\infty}}{1-q^{n}}\right)
$$




$$
=b_{n+1}(f * g)+\sum_{s= \pm 1} \sum_{j=1}^{\left\lfloor\frac{\sqrt{24 k+25}-s}{6}\right\rfloor}(-1)^{j} b_{n+1-\frac{j(3 j+s)}{2}}(f * g),
$$

where $b_{k}(f * g)=\sum_{d \mid n}(f * g)(d)$ is as on the left-hand-side of $(1)$ for $(\alpha, \beta):=(1,0)$. Then using the notation in Proposition 4.1 and in the claim immediately above, we see that our new expansions imply that

$$
f(n)=\sum_{k=1}^{n} s_{n, k}^{(-1)}(g) \cdot B_{k-1}(f, g) .
$$

We will employ several well-known Dirichlet convolution results which provide applications of the previous expansions. For example, given any fixed $t \in \mathbb{C}$ we have the known identity that $\operatorname{Id}_{t}=\sigma_{t} * \mu$ where $\operatorname{Id}_{t}(n)=n^{t}$ is the $t^{t h}$ power function. In particular, this identity implies each of the following identities:

$$
\begin{aligned}
\mu(n) & =\sum_{k=1}^{n} s_{n, k}^{(-1)}\left(\sigma_{t}\right) \cdot B_{k-1}\left(\sigma_{t}\right) \\
\sigma_{t}(n) & =\sum_{k=1}^{n} s_{n, k}^{(-1)}(\mu) \cdot B_{k-1}\left(\sigma_{t}\right) .
\end{aligned}
$$

Similarly, since $\operatorname{Id}_{1}=\phi * 1, \sigma_{1}=\phi * \operatorname{Id}_{1}$, and $\Lambda=\log * \mu$, we have the following related expansions:

$$
\begin{aligned}
\phi(n) & =\sum_{k=1}^{n} s_{n, k}^{(-1)}\left(\operatorname{Id}_{0}\right) \cdot B_{k-1}\left(\sigma_{1}\right) \\
1 & =\sum_{k=1}^{n} s_{n, k}^{(-1)}(\phi) \cdot B_{k-1}\left(\sigma_{1}\right) \\
\phi(n) & =\sum_{k=1}^{n} s_{n, k}^{(-1)}\left(\operatorname{Id}_{1}\right) \cdot B_{k-1}\left(\sigma_{1} * 1\right) \\
n & =\sum_{k=1}^{n} s_{n, k}^{(-1)}(\phi) \cdot B_{k-1}\left(\sigma_{1} * 1\right) \\
\log (n) & =\sum_{k=1}^{n} s_{n, k}^{(-1)}(\mu) \cdot B_{k-1}(\log ) \\
\mu(n) & =\sum_{k=1}^{n} s_{n, k}^{(-1)}(\log ) \cdot B_{k-1}(\log ) .
\end{aligned}
$$

We emphasize that the formulas listed above are special in nature due to the unconventional dependence of the inverse sequences, $s_{n, k}^{(-1)}(g)$ on multiple $j$-fold convolutions of the function $g$ for $1 \leq j \leq n$. The next explicit computation illustrates this property for the identity tagged in $(\dagger)$ above when $n:=4$ :

$$
\begin{aligned}
1= & {\left[\left(\phi_{ \pm} * \phi * \phi * \phi\right)(4)-3 \cdot\left(\phi_{ \pm} * \phi * \phi\right)(4)+4 \cdot\left(\phi_{ \pm} * \phi\right)(4)-2 \cdot \phi(4)+2\right] \sigma(1) } \\
& -\left[\left(\phi_{ \pm} * \phi\right)(2)-\phi(2)+1\right](\sigma(1)-\sigma(2))-(\sigma(1)+\sigma(2)-\sigma(3)) \\
& -(\sigma(2)+\sigma(3)-\sigma(4)) .
\end{aligned}
$$


Many other examples of known convolution results to which we can apply our new expansions are found in the references $[1,3]$.

Corollary 4.4 (Formulas for the Dirichlet Inverse). For any prescribed arithmetic function $f$ defined such that $f(1)=1$, we have a formula for its Dirichlet inverse function given by

$$
f^{-1}(n)=\sum_{k=1}^{n}\left(\left(p_{k} * \mu\right)(n)+\left(p_{k} * D_{n, f} * \mu\right)(n)\right) \cdot\left[q^{k-1}\right] \frac{(q ; q)_{\infty}}{1-q} .
$$

Proof. The proof follows from Theorem 4.2 applied in the form of Proposition 4.1. In particular, since $f^{-1} * f=\delta_{n, 1}$ by definition, the right-hand-side of our Lambert series expansion over the convolved function $a_{n}:=f^{-1} * f$ is given by $q /(1-q)$.

We compare this formula for the Dirichlet inverse of an arithmetic function to the other primary known recursive divisor sum formula defining the inverse function of $f$ given by $[1, \S 2.7]$

$$
f^{-1}(1)=\frac{1}{f(1)}, f^{-1}(n)=-\frac{1}{f(1)} \sum_{\substack{d \mid n \\ d<n}} f(n / d) f^{-1}(d) \text { for } n>1,
$$

for $f(1) \neq 0$ and where it is known that $(f * g)^{-1}=f^{-1} * g^{-1}$ if $f(1), g(1) \neq 0$. The Dirichlet inverse of a function $f$ exists precisely when $f(1) \neq 0$, and by scaling our result in the corollary matches these cases as well.

We also note that given any prescribed sequence of $b(n)$ we can generate $b(n)$ by the Lambert series over $b * \mu$. This implies that we have recurrence relations for any arithmetic function $b$ defined such that $b(n)=0$ for all $n<0$ expanded in the following two forms where $s_{n, k}:=\left[q^{n}\right](q ; q)_{\infty} q^{k} /\left(1-q^{k}\right)$ :

$$
\begin{aligned}
& b(n)=\sum_{k=1}^{n}\left(p_{k} * \mu+p_{k} * D_{n, \mu} * \mu\right)(n)\left[b(k)+\sum_{s= \pm 1} \sum_{j=1}^{k}(-1)^{j} b\left(k-\frac{j(3 j+s)}{2}\right)\right] \\
& b(n)=\sum_{j=1}^{n} \sum_{k=1}^{j}\left(\sum_{i=1}^{\lfloor j / k\rfloor} s_{j, k i} \cdot \mu(i)\right) b(k) p(n-j) .
\end{aligned}
$$

Corollary 4.5 (Convolution Formulas for Arithmetic Functions). Suppose that we have two prescribed arithmetic functions $f$ and $h$ and we seek the form of a third $g$ satisfying $f * g=h * \mu$ for all $n \geq 1$. Then we have a formula for the function $g$ expanded in the form of

$$
\begin{aligned}
g(n)=\sum_{k=1}^{n} & \left(\left(p_{k} * \mu\right)(n)+\left(p_{k} * D_{n, f} * \mu\right)(n)\right) \times \\
& \times\left(h(k)+\sum_{s= \pm 1} \sum_{j=1}^{\left.\frac{\sqrt{24 k+1}-s}{6}\right\rfloor}(-1)^{j} h\left(k-\frac{j(3 j+s)}{2}\right)\right) .
\end{aligned}
$$

Proof. This result is an immediate consequence of Proposition 4.1 and the formula for the inverse sequences defined by Theorem 4.2. 


\section{Conclusions}

5.1. Summary. In Section 2 and Section 3 we proved two key new variations of the Lambert series factorization theorems studied in the references $[4,5,6,8]$ each of which effectively generalize the expansions of these initial model results to the more general cases of the Lambert series expansions defined by (1) in the introduction. The propositions for special cases and the examples discussed in Section 2.1 motivate the formulation of Theorem 2.5 which provides generating functions for the factorization parameters, $s_{n, k}$, in the most general cases and which motivate its immediate consequence stated in Corollary 2.6 providing the connection between our new generalized factorization theorems and the known ordinary cases established by the references.

We have similarly proved a variation of the generalized factorization theorem stated by Theorem 3.1 in Section 3 along the lines of the same motivations for the corresponding "ordinary" case result in [5]. That is, instead of starting with the right-hand-side factorizations in (3), we define the form of $s_{n, k}^{(-1)}$ which determines the entries of the matrix of $s_{n, k}$ and then proceed to explore the form of the resulting factorizations based on our definition. It turns out that the formulas in this case provide a natural analog to the corresponding result for the Lambert series cases expanded in the reference.

The truly new results unique to this article are stated and proved in Section 4 where we provide a variant of the Lambert series factorization theorems given in the previous sections and in the references for a convolution of two arithmetic functions. These new results lead to applications for several well-known Dirichlet convolutions of classical arithmetic functions which are expanded by multiple $j$-fold convolutions of one of these classical functions with itself.

5.2. A note on further generalizations. It is possible to consider even more general factorization theorem results for Lambert series expansions of the form

$$
L_{a}(\alpha, \beta ; c, d ; q):=\sum_{n \geq 1} \frac{a_{n} c^{n} q^{\alpha n-\beta}}{1-d \cdot q^{\alpha n-\beta}},
$$

for $\alpha, \beta$ defined in the conventions stated in the introduction and for some fixed constants $c, d \in \mathbb{C}$ defined such that the series in the previous equation converges when $\left|q^{\alpha}\right|<1$, though for the most part we do not consider such expansions here. We do however motivate these expansions when $c:=1$ in the second degenerate case of Theorem 2.5 discussed in Remark 2.7 by demonstrating the similarities and relations to the corresponding ordinary degenerate case of this series where $d \equiv \pm 1$.

Except in a few rare cases of Lambert series expansions related to special functions where $d:=-1$, the consideration of the additional parameters $c, d$ does not seem to add much utility to phrasing our notable new identities for the classical special functions. We also note that in this particular case we have a transformation identity for the ordinary cases of the Lambert series expansions in (1) of the form $[5]$

$$
\begin{aligned}
\sum_{n=1}^{\infty} \frac{a_{n} q^{n}}{1+q^{n}} & =\sum_{n=1}^{\infty} \frac{a_{n} q^{n}}{1-q^{n}}-2 \sum_{n=1}^{\infty} \frac{a_{n} q^{2 n}}{1-q^{2 n}} \\
& =\sum_{n=1}^{\infty} \frac{b_{n} q^{n}}{1-q^{n}},
\end{aligned}
$$


where

$$
b_{n}= \begin{cases}a_{n}, & \text { for } n \text { odd } \\ a_{n}-2 a_{n / 2} & \text { for } n \text { even. }\end{cases}
$$

We therefore leave such generalizations as a topic suggested for future work separate from the results we have established within this article.

5.3. Other Variants of the Factorization Theorems. There are many other variations of the Lambert series factorization theorems we have proved in Proposition 4.1 and in the previous sections whose properties we have still left yet unexplored. As an example of what other useful factorizations are possible, for any fixed arithmetic function $a_{n}$ let $A(x):=\sum_{n \leq x} a_{n}$. Then we may consider the properties, i.e., inverses, etc., of the factorizations of the following forms, among several other possibilities:

$$
\begin{aligned}
& \sum_{n \geq 1} \frac{A(n) q^{n}}{1-q^{n}}=\frac{1}{(q ; q)_{\infty}} \sum_{n \geq 1} \sum_{k=1}^{n} s_{1, n, k} \cdot a_{k} \cdot q^{n} \\
& \sum_{n \geq 1} \frac{a_{n} q^{n}}{1-q^{n}}=\frac{1}{(q ; q)_{\infty}} \sum_{n \geq 1} \sum_{k=1}^{n} s_{2, n, k} \cdot A(k) \cdot q^{n} .
\end{aligned}
$$

The factorization expanded in the second of the previous equations has the benefit that to analyze the average order $A(x)$ of the prescribed arithmetic function it is only necessary to examine the properties of the sequence of inverses, $s_{2, n, k}^{(-1)}$, and the form of the function $a_{n} * 1$. Then using the method of proof in Theorem 2.5, we can show that

$$
s_{1, n, k}=d(n)+\sum_{s= \pm 1} \sum_{j=1}^{\left\lfloor\frac{\sqrt{24 k+1}-s}{6}\right\rfloor}(-1)^{j} d\left(n-\frac{j(3 j+s)}{2}\right)-\sum_{i=1}^{k-1} s_{n, i}
$$

and that

$$
s_{n, k}=\sum_{i=0}^{n-k} s_{2, n, n-i} \quad \text { and } \quad s_{2, n, k}=s_{n, k}-s_{n, k+1},
$$

where $s_{n, k}=\left[q^{n}\right] q^{k} /\left(1-q^{k}\right) \cdot(q ; q)_{\infty}$ denotes the difference of the number of $k$ 's in all partitions of $n$ into an odd number of distinct parts and into an even number of parts. These identities also imply that

$$
(A * 1)(n)=\sum_{k=1}^{n} s_{1, n, k} \cdot a_{k} \quad \text { and } \quad(a * 1)(n)=\sum_{k=1}^{n} s_{2, n, k} \cdot A(k),
$$

for any prescribed arithmetic function $a_{n}$. Additionally, we can show that

$$
\begin{aligned}
s_{1, n, k}^{(-1)} & =s_{n, k}-s_{n-1, k}[n>1]_{\delta} \\
& =\sum_{d \mid n} p(d-k) \mu(n / d)-\sum_{d \mid n-1} p(d-k) \mu((n-1) / d)[n>1]_{\delta} \\
s_{2, n, k}^{(-1)} & =\sum_{j=1}^{n} s_{j, k}=\sum_{j=1}^{n} \sum_{d \mid j} p(d-k) \mu(j / d),
\end{aligned}
$$


which then implies new exact identities such as the following expansions involving $a_{n}$ for $n \geq 2$ :

$$
\begin{aligned}
a_{n} & =\sum_{k=1}^{n} \sum_{s \in\{0,1\}} \sum_{d \mid n-s}(-1)^{s} p(d-k) \mu\left(\frac{n-s}{d}\right) \cdot\left[q^{k}\right]\left(\sum_{m \geq 1}(A * 1)(m) q^{m} \times(q ; q)_{\infty}\right) \\
A(n) & =\sum_{k=1}^{n} \sum_{j=1}^{n} \sum_{d \mid j} p(d-k) \mu(j / d) \cdot\left[q^{k}\right]\left(\sum_{m \geq 1}(a * 1)(m) q^{m} \times(q ; q)_{\infty}\right) .
\end{aligned}
$$

Some properties of the generalized cases of these factorization theorems are apparent by inspection of the examples cited above. In particular, suppose that we have factorizations of the form

$$
\begin{aligned}
& \sum_{n \geq 1} \frac{a_{n} q^{n}}{1-q^{n}}=\frac{1}{C(q)} \sum_{n \geq 1} \sum_{k=1}^{n} s_{n, k} \cdot a_{k} \cdot q^{n} \\
& \sum_{n \geq 1} \frac{a_{n} q^{n}}{1-q^{n}}=\frac{1}{C(q)} \sum_{n \geq 1} \sum_{k=1}^{n} \widetilde{s}_{n, k}\left(\sum_{i=1}^{k} b_{i} a_{i}\right) \cdot q^{n},
\end{aligned}
$$

for some sequence $b_{i}$ of non-zero functions. Then it is not difficult to prove that

$$
\widetilde{s}_{n, k}=\frac{s_{n, k}}{b_{k}}-\frac{s_{n, k+1}}{b_{k+1}} \quad \text { and } \quad \widetilde{s}_{n, k}^{(-1)}=\sum_{i=1}^{n} b_{i} \cdot s_{i, k}^{(-1)} .
$$

To give some additional possibilities for factorization theorems to consider based on the results in this article and in the references, we note that we may expand

$$
\begin{aligned}
\sum_{n \geq 1} \frac{a_{n} q^{n}}{1-q^{n}} & =\frac{1}{C(q)} \sum_{n \geq 1} \sum_{k=1}^{n} s_{n, k}(a) \cdot a_{k}^{-1} \cdot q^{n} \\
\sum_{n \geq 1} \frac{A(n) q^{n}}{1-q^{n}} & =\frac{1}{C(q)} \sum_{n \geq 1} \sum_{k=1}^{n} s_{n, k}(a)\left(\sum_{i=1}^{k} \frac{a_{i}}{i}\right) \cdot q^{n}
\end{aligned}
$$

where $a_{n}^{-1}$ is the Dirichlet inverse of $a_{n}$, and that we may expand

$$
\sum_{n \geq 1} \frac{\widetilde{a}_{n} q^{n}}{1-q^{n}}=\frac{1}{C(q)} \sum_{n \geq 1} \sum_{k=1}^{n} s_{n, k}(\gamma) \cdot A(k) \cdot q^{n}
$$

where we define $s_{n, k}(\gamma)$ implicitly by $s_{n, k}^{(-1)}:=\sum_{d \mid n}\left[q^{d-k}\right] \frac{1}{C(q)} \cdot \gamma(n / d)$ and where we conjecture that the function $\widetilde{a}_{n}$ is defined explicitly as the following sum involving the $A(k)$ and the $j$-fold convolution functions $D_{n, \gamma}(k)$ from Section 4:

$$
\widetilde{a_{n}}=\sum_{d \mid n} \sum_{\substack{r \mid d \\ r>1}} A(n / d) D_{n, \gamma}(r) \mu(d / r)+\sum_{d \mid n} A(d) \mu(n / d) .
$$

For the most part, except for the remarks given above in this subsection, we leave these and other expansions of analogous factorization theorems as a topic of future research based on the work in this article and in the references $[4,5,6,8]$.

Acknowledgments. The authors thank the referees for their helpful insights and comments on preparing the manuscript. 


\section{REFERENCES}

[1] T. M. Apostol, Introduction to analytic number theory, Springer, 1976.

[2] G. H. Hardy and E. M. Wright. An Introduction to the Theory of Numbers. Oxford University Press, 2008

[3] R. J. Mathar, Survey of Dirichlet series of multiplicative arithmetic functions, 2012, https://arxiv.org/abs/1106.4038.

[4] M. Merca and M. D. Schmidt, Generating special arithmetic functions by Lambert series factorizations, 2017, https://arxiv.org/abs/1706.00393, submitted.

[5] M. Merca and M. D. Schmidt, New Factor Pairs for Factorizations of Lambert Series Generating Functions, 2017, https://arxiv.org/abs/1706.02359, submitted.

[6] M. Merca, The Lambert series factorization theorem, Ramanujan J., pp. 1-19 (2017).

[7] F. W. J. Olver, D. W. Lozier, R. F. Boisvert, and C. W. Clark. NIST Handbook of Mathematical Functions. Cambridge University Press, 2010.

[8] M. D. Schmidt, New recurrence relations and matrix equations for arithmetic functions generated by Lambert series, 2017, https://arxiv.org/abs/1701.06257. Tentatively accepted for publication in Acta Arith..

[9] N. J. A. Sloane, The Online Encyclopedia of Integer Sequences, 2017, https://oeis.org/. 